\title{
Formal preparation of regioregular and alternating thiophene-thiophene copolymers bearing different substituents
}

\author{
Atsunori Mori ${ }^{*} 1,2$, Keisuke Fujita ${ }^{1}$, Chihiro Kubota ${ }^{1}$, Toyoko Suzuki ${ }^{1}$, Kentaro Okano ${ }^{1}$, \\ Takuya Matsumoto ${ }^{1}$, Takashi Nishino ${ }^{1}$ and Masaki Horie ${ }^{3}$
}

\author{
Full Research Paper \\ Address: \\ ${ }^{1}$ Department of Chemical Science and Engineering, Kobe University, \\ 1-1 Rokkodai, Nada, Kobe 657-8501, Japan, ${ }^{2}$ Research Center for \\ Membrane and Film Technology, Kobe University, 1-1 Rokkodai, \\ Nada, Kobe 657-8501, Japan and ${ }^{3}$ Department of Chemical \\ Engineering, National Tsing Hua University, 101, Sec. 2, Kuang-Fu \\ Road, Hsinchu 30013, Taiwan \\ Email: \\ Atsunori Mori ${ }^{*}$ - amori@kobe-u.ac.jp \\ * Corresponding author

\section{Keywords:} \\ alternating copolymer; nickel(II) catalyst; oligosiloxane; regioregular \\ polythiophene; solubility
}

Beilstein J. Org. Chem. 2020, 16, 317-324.

doi:10.3762/bjoc. 16.31

Received: 27 December 2019

Accepted: 26 February 2020

Published: 05 March 2020

This article is part of the thematic issue " $\mathrm{C}-\mathrm{H}$ functionalization for materials science".

Guest Editor: K. Itami

(C) 2020 Mori et al.; licensee Beilstein-Institut.

License and terms: see end of document.

\begin{abstract}
Differently substituted thiophene-thiophene-alternating copolymers were formally synthesized employing a halo-bithiophene as a monomer. Nickel-catalyzed polymerization of bithiophene with substituents at the 3-position, including alkyl-, fluoroalkyl-, or oligosiloxane-containing groups, afforded the corresponding copolymers in good to excellent yield. The solubility test in organic solvents was performed to reveal that several copolymers showed a superior solubility. X-ray diffraction analysis of the thin film of the alternating copolymers composed of methyl and branched oligosiloxane substituents was also performed, and the results suggested the formation of a dual-layered film structure.
\end{abstract}

\section{Introduction}

Polythiophenes attract much attention in materials science because of their extended $\pi$-conjugation, which is applied for a wide range of electronic materials. In particular, the regioregular polymers with a head-to-tail (HT) orientation with respect to the substituent at the 3-position are extensively studied to date since they generally show superior performances as materials [1-6]. Cross-coupling polymerization catalyzed by a transition metal complex has been recognized as an effective tool to afford the regioregular polythiophene in which 2,5-dihalo-3substituted thiophene $\mathbf{1}$ is employed as a monomer precursor, 
converting to the corresponding organometallic monomer by a halogen-magnesium exchange reaction with a Grignard reagent. The employment of $\mathbf{1}$ leading to polythiophene has been shown to proceed in a dehalogenative manner [3]. We have recently shown that the generation of the organometallic monomer species can alternatively also be achieved by deprotonation, using 2-halo-3-substituted thiophene $\mathbf{2}$ or $\mathbf{3}$ with a bulky magnesium amide Knochel-Hauser base (TMPMgCl.LiCl) [7], followed by polymerization catalyzed by a nickel complex, leading to the regioregular HT polythiophene (Scheme 1) [8,9]. An additional feature of the deprotonative protocol for polythiophene is the possibility to use chlorothiophene 3 , in which the use of a nickel N-heterocyclic carbene (NHC) complex was found to be effective $[10,11]$. We have also engaged in the design of the side chain of polythiophenes, and several functionalities have been successfully introduced
[12-14]. We further focused on the copolymerization of thiophene, employing differently substituted thiophene monomers, with which several copolymerizations are plausible, giving thiophene-thiophene copolymers of random (statistical) [15], gradient [16,17], block [18,19], alternating [20-23], etc. [24,25] makeup. We are thus interested in the preparation of alternating polythiophenes bearing two kinds of different substituents. We envisaged that such an alternating copolymer in perfect regularity can be achieved by deprotonative polymerization employing a bithiophene with different substituents at the 3- and 3'-position.

We have recently shown that the coupling of 2-chloro-3-substituted thiophene 2 with 2-bromo-3-substituted thiophene 3 efficiently gave chlorobithiophene $\mathbf{4}$ in a facile manner (Scheme 2) [25]. The use of a palladium catalyst efficiently suppressed the

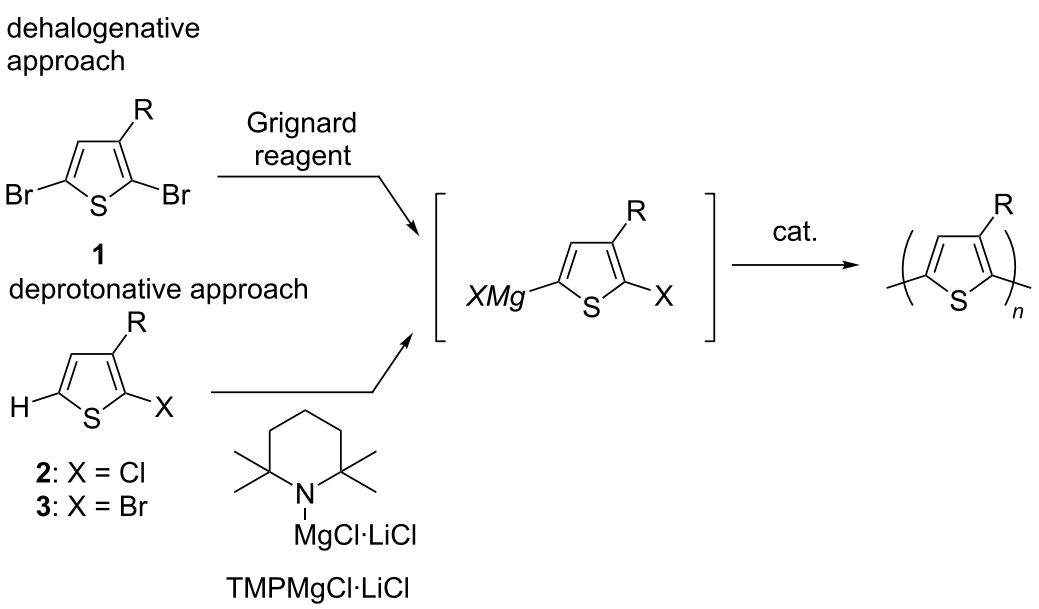

Scheme 1: Cross-coupling polymerization of thiophene.

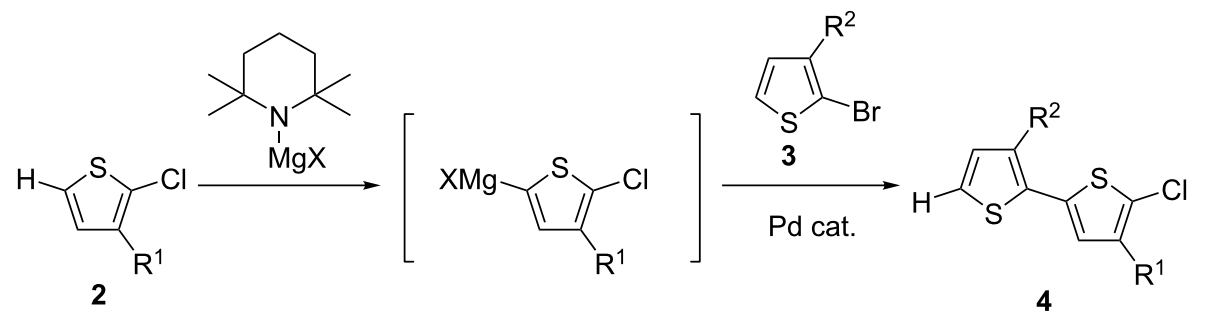

4

Ni cat.

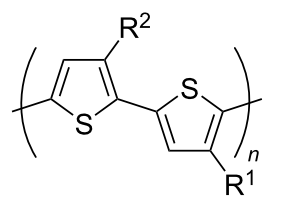

alternating copolymer 
undesired polymerization to afford the HT halobithiophene with different substituents [26]. Compared to an alternative pathway to 4 , in which initial coupling is followed by chlorination, this protocol exploits the improved deprotonation efficiency of $\mathbf{2}$ toward 3'-unsubstituted 3-substituted bithiophene, and this method enabled the synthesis of 4 (where $R^{1}=H$ ) regioselectively. Polymerization of 4 (where $\mathrm{R}^{1}=n$-hexyl and $\mathrm{R}^{2}=$ $\left.\left(\mathrm{CH}_{2}\right)_{4} \mathrm{Si}\left(\mathrm{Me}_{2}\right) \mathrm{OSiMe}_{3}\right)$ was also examined preliminarily, and it was confirmed that the alternating copolymer was obtained with extremely high regularity. Herein, we wished to study the polymerization of bithiophene $\mathbf{4}$ possessing several kinds of substituents and a variety of functionalities. Since the homopolymer is considered rather insoluble in most organic solvents, the presumed improved solubility of the related alternating copolymers was also part of this study.

\section{Results and Discussion}

The synthesis of chlorobithiophenes with different substituents at the 3- and 3'-position was carried out in a manner that we have described previously [25]. We chose five chlorobithiophenes as monomer precursors for the alternating copolymers, as summarized in Scheme 3. The cross-couplings, as shown in Scheme 2, proceeded smoothly to afford the bithiophenes 4 in $46-92 \%$ yield.

The synthesis of the alternating copolymers was carried out with monomer precursors $\mathbf{4}$ by deprotonation with Knochel-Hauser base followed by the addition of the nickel catalyst $\mathrm{NiCl}_{2}\left(\mathrm{PPh}_{3}\right)$ IPr to initiate the polymerization of bithiophene.

We first carried out the polymerization of chlorobithiophene $\mathbf{4 a}$, bearing hexyl and methyl substituents at the 3- and 3'-position, respectively. Although the polymerization took place in moderate yield (34\%), the formation of hardly soluble precipitates was observed during the reaction, and the thus obtained solid was found to fail to dissolve in any organic solvent. As previously reported for the regioregular polythiophene synthesis, poly(3-hexylthiophene) (P3HT) can smoothly be dissolved in several organic solvents. In contrast, there have been few reports on the preparation of regioregular polythiophene bearing a methyl group at the 3-position [27]. Accordingly, the incorporation of the alternating methyl substituent would result in much inferior solubility as compared to the alternating copolymer. Several chlorobithiophenes 4 were then similarly subjected to the polymerization. The results of the alternating copolymerizations are summarized in Scheme 4. The deprotonation by the Knochel-Hauser base was carried out at room temperature for $3 \mathrm{~h}$. The addition of the nickel catalyst 5 and further stirring at room temperature followed. The reactions proceeded smoothly to afford the corresponding formally alternating copolymers in $48-84 \%$ yields [28]. The molecular weight of the products was found to be controllable based on the ratio of monomer/catalyst feed, and the molecular weight distributions were relatively narrow.

Solubility tests of the obtained polymers were performed as summarized in Figure 1. Although the alternating copolymer 6b, substituted with methyl and C-4 alkyl groups as well as terminal pentamethyldisiloxane groups, was obtained with a slightly low molecular weight, suggesting improved solubility compared to $\mathbf{6 a}\left(\mathrm{R}^{2}=\mathrm{Me}\right.$ and $\mathrm{R}^{1}=n$-hexyl), the attempted dissolution of the obtained polythiophene $\mathbf{6} \mathbf{b}$ in chloroform was unsuccessful. Switching the oligosiloxane moiety to a branched derivative $\left(\mathrm{R}^{2}=\left(\mathrm{CH}_{2}\right)_{4} \mathrm{Si}(\mathrm{Me})\left(\mathrm{OSiMe}_{3}\right)_{2}\right)$ in $\mathbf{6 c}$ remarkably improved the solubility, and the copolymer $\mathbf{6 c}$ was soluble

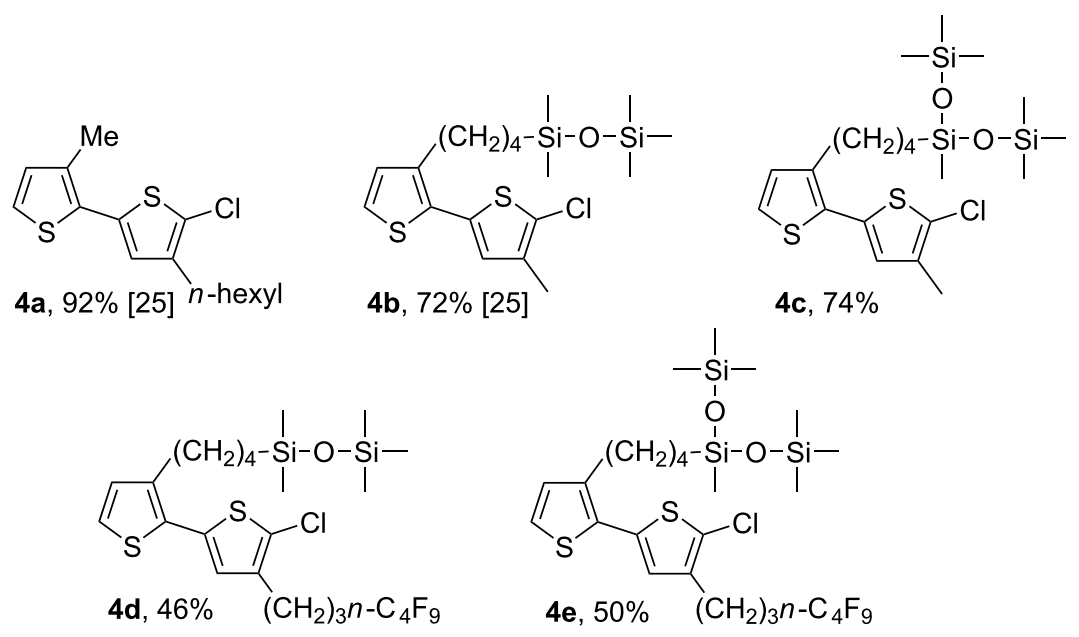

Scheme 3: Preparation of chlorobithiophenes. 


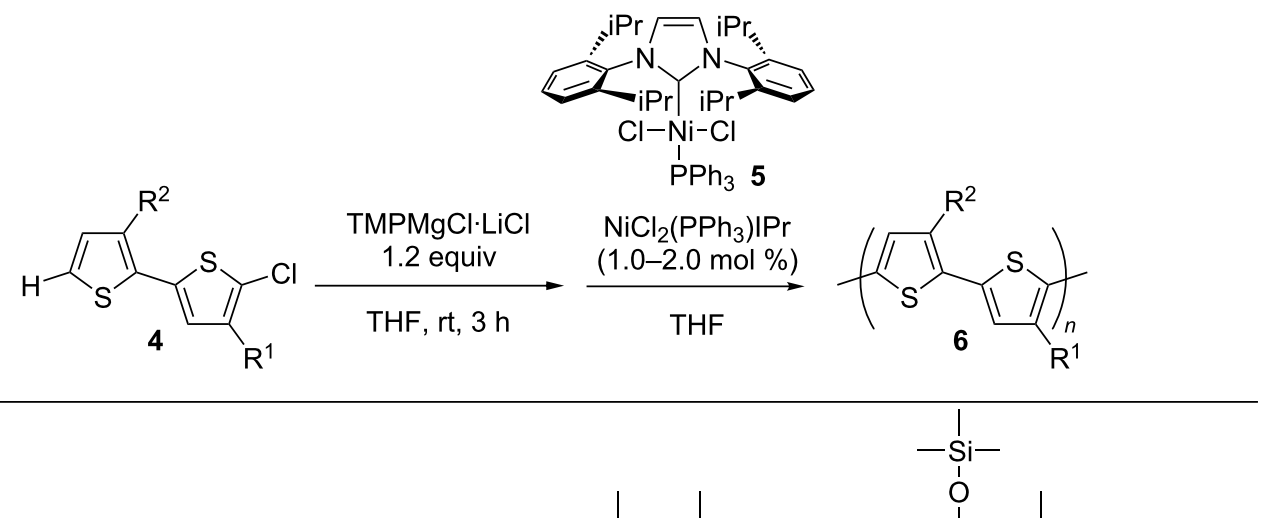

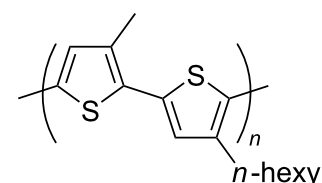

6a, $34 \%$ $(2.0 \mathrm{~mol} \%$ $60{ }^{\circ} \mathrm{C}, 20 \mathrm{~h}$ ) low solubility

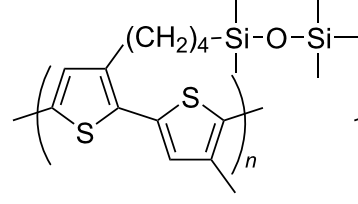

6b, $84 \%$

(2.0 mol \%, rt, $24 \mathrm{~h})$ $M_{\mathrm{n}}=10400, M_{\mathrm{w}} / M_{\mathrm{n}}=1.62$

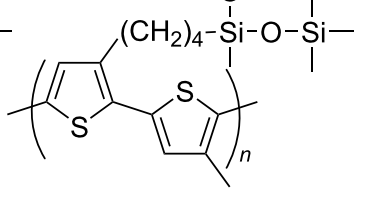

6c, $84 \%$

$(1.0 \mathrm{~mol} \%, \mathrm{rt}, 18 \mathrm{~h})$

$M_{\mathrm{n}}=70100, M_{\mathrm{w}} / M_{\mathrm{n}}=1.38$<smiles>Cc1cc(C)c(-c2cc(C)c(CC(C)(C)O[Si](C)(O[Si](C)(C)C)O[Si](C)(C)C)s2)s1</smiles>

$6 \mathrm{~d}, 48 \%$

(1.0 mol \%, rt, $1 \mathrm{~h}$ ) $M_{\mathrm{n}}=68600, M_{\mathrm{w}} / M_{\mathrm{n}}=1.15$

$6 e, 58 \%$

$(1.0 \mathrm{~mol} \%, \mathrm{rt}, 1 \mathrm{~h})$

$M_{\mathrm{n}}=33200, M_{\mathrm{w}} / M_{\mathrm{n}}=1.07$

Scheme 4: Polymerization of chlorobithiophenes.

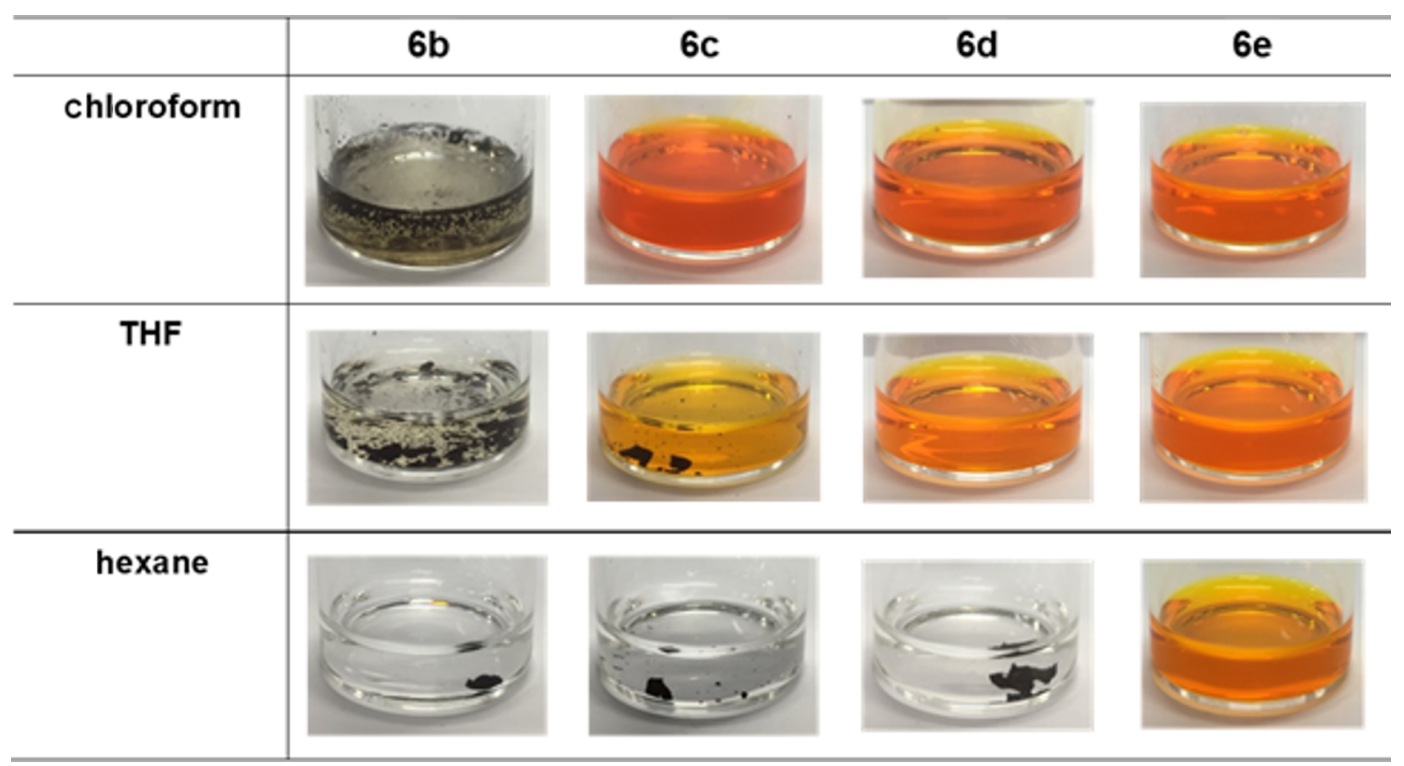

Figure 1: Solubility tests of alternating copolymer 6 (1 mg of material dissolved in $1 \mathrm{~mL}$ of the solvent). 
in chloroform, whereas the dissolution in hexane was unsuccessful. Copolymers bearing a fluoroalkyl substituent $\left(\left(\mathrm{CH}_{2}\right)_{3} n-\mathrm{C}_{4} \mathrm{~F}_{9}\right)$, with a corresponding homopolymer solubility that was lower than that of the long-chained alkyl derivatives, were then examined. The alternating copolymer $\mathbf{6 d}$, bearing fluoroalkyl and non-branched disiloxane substituents was readily soluble in chloroform, while the attempted dissolution of $\mathbf{6 d}$ in hexanes was unsuccessful. However, a remarkable solubility of the copolymer $\mathbf{6 e}$ bearing a fluoroalkyl group and a branched oligosiloxane unit in hexanes was noted.

XRD analysis of the copolymer $\mathbf{6 c}$, bearing branched oligosiloxane and methyl groups, was carried out. Two remarkable peaks were observed at $2 \theta=3.94^{\circ}$ and $12.18^{\circ}$, respectively, as shown in Figure 2a. The result suggested that the thin film of the alternating copolymer $\mathbf{6 c}$ had a bilayer lamellar structure with $7.3 \AA$ and $22.4 \AA$ distances, respectively (Figure 2b) $[13,29,30]$. Molecular modeling of the alternating copolymer $\mathbf{6 c}$ suggested chain lengths of $11.6 \AA$ and $2.2 \AA$, respectively. The values of the observed layer distances of copolymer $\mathbf{6 c}$ closely corresponded to twice the values, $11.6 \times 2$ and $2.2 \times 2$ (Figure 2c), in which the conformation of the carbon-carbon single bond between the thiophene rings was anti. The proposed layer distance of regiorandom poly(3-methylthiophene) of $7.7 \AA$ reported by Yan and co-workers was close to our result from the XRD analysis (7.3 $\AA$ ) corresponding to the aggregation of the alternating methyl substituent [27].

\section{Conclusion}

In summary, we showed that formally alternating thiophene-thiophene copolymers could be synthesized by employing differently substituted halobithiophenes as monomers in nickel-catalyzed deprotonative polymerizations. The introduction of oligosiloxanes as side chains improved the solubility in organic solvents, and copolymer components involving less soluble functional groups, such as short alkyl chains or fluoroalkyl groups, could be incorporated into the alternating copolymers. X-ray diffraction measurements revealed that the alternating copolymers bearing different side chain lengths had dual-layer structures in the thin-film state.

\section{Experimental General}

Polymerization was carried out with the standard Schlenk technique under a nitrogen or argon atmosphere. ${ }^{1} \mathrm{H}$ NMR (400 MHz), ${ }^{19} \mathrm{~F}$ NMR (376 MHz), and ${ }^{13} \mathrm{C}\left\{{ }^{1} \mathrm{H}\right\}$ NMR $(100 \mathrm{MHz})$ spectra were measured on a JEOL ECZ400 spectrometer as a $\mathrm{CDCl}_{3}$ solution, unless otherwise noted. The chemical shifts were expressed in ppm with $\mathrm{CHCl}_{3}(7.26 \mathrm{ppm}$ for $\left.{ }^{1} \mathrm{H}\right), \mathrm{C}_{6} \mathrm{~F}_{6}\left(-164.9 \mathrm{ppm}\right.$ for $\left.{ }^{19} \mathrm{~F}\right)$, or $\mathrm{CDCl}_{3}$ (77.16 ppm for $\left.{ }^{13} \mathrm{C}\right)$ as internal standards. IR spectra were recorded on Bruker Alpha spectrometer with an ATR attachment (Ge). High-resolution mass spectra (HRMS) were measured using a JEOL JMST100LP AccuTOF LC-Plus apparatus (ESI) with a JEOL MS-5414DART attachment. For thin-layer chromatography (TLC) analyses throughout this work, Merck precoated TLC plates (silica gel $60 \mathrm{~F}_{254}$ ) were used. Purification by HPLC with preparative SEC columns (JAI-GEL-1H and JAI-GEL-2H) was performed by a JAI LC-9201 system. SEC analyses were carried out with a standard HPLC system equipped with a UV detector at $40{ }^{\circ} \mathrm{C}$ using $\mathrm{CHCl}_{3}$ as an eluent with Shodex KF-402HQ and KF-404HQ. Molecular weights and molecular weight distributions were estimated on the basis of the calibration curve obtained by 6 standard polystyrenes. UV-vis absorption spectra of the polymer films were measured with a Shimadzu UV-3150 spectrometer. XRD analysis was carried out with a Rigaku RINT-2000(CuK $\alpha)$ device. Concerning the solvent of the nickel- and palladium-catalyzed reactions, THF (anhydrous grade) was purchased from Kanto Chemical Co. Ltd. and passed through alumina and copper columns (Nikko
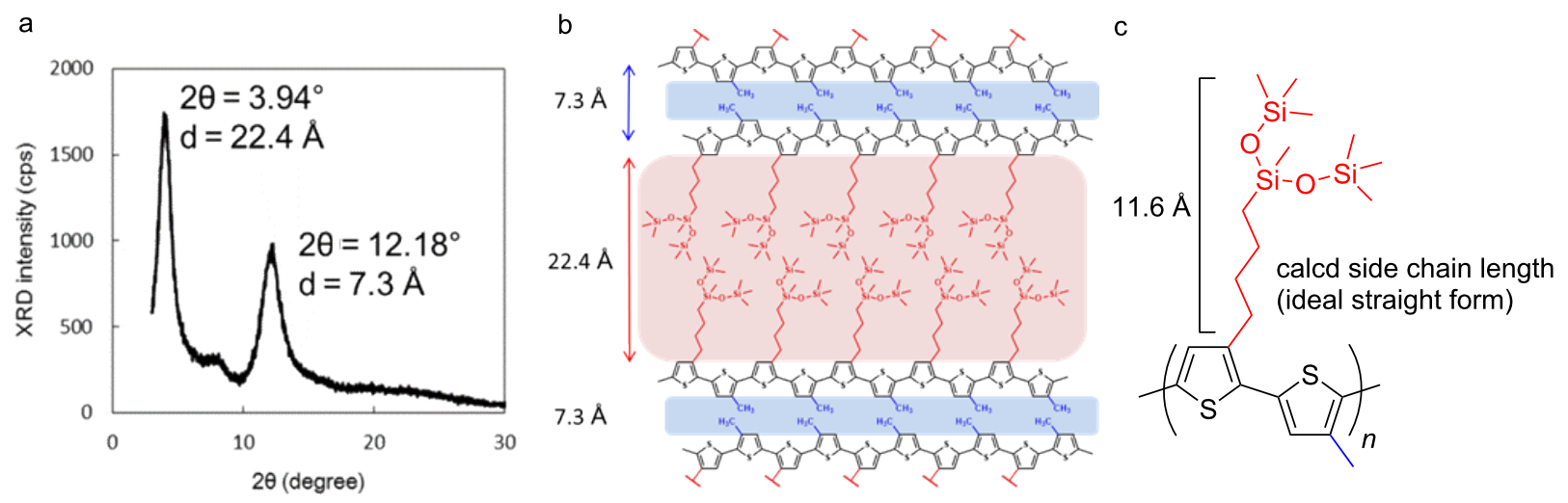

Figure 2: XRD measurement and prediction of the bilayer lamellar structure of polymer 6c. a) XRD analysis. b) Suggested bilayer lamellar structure. c) Side chain length. 
Hansen \& Co. Ltd.) or distilled from a sodium dispersion in mineral oil/benzophenone ketyl [31] prior to use. The Knochel-Hauser base (TMPMgCl-LiCl) was purchased from Sigma-Aldrich Co. Ltd. as a $1 \mathrm{M}$ THF solution. $\mathrm{NiCl}_{2}\left(\mathrm{PPh}_{3}\right) \mathrm{IPr}$ (5) was purchased from TCI Co. Ltd. All other chemicals were purchased and used without further purification. The preparation of the chlorobithiophenes $\mathbf{4 a}$ and $\mathbf{4 b}$ was performed in a manner reported previously [25].

The synthesis of the chlorobithiophenes $\mathbf{4}$ was carried out in a manner shown in our previous report [25]. The spectroscopic properties and analytical data for $\mathbf{4}$ are summarized below.

2-Chloro-3-hexyl-5-(3-methylthiophen-2-yl)thiophene (4a) [25]: $92 \%$ yield as a light yellow oil. ${ }^{1} \mathrm{H}$ NMR $(500 \mathrm{MHz}$, $\left.\mathrm{CDCl}_{3}\right) \delta 0.92(\mathrm{t}, J=7.5 \mathrm{~Hz}, 3 \mathrm{H}), 1.31-1.43(\mathrm{~m}, 6 \mathrm{H})$, $1.58-1.67(\mathrm{~m}, 2 \mathrm{H}), 2.37$ (s, 3H), 2.59 (t, $J=7.5 \mathrm{~Hz}, 2 \mathrm{H}), 6.83$ $(\mathrm{s}, 1 \mathrm{H}), 6.87(\mathrm{~d}, J=5.0 \mathrm{~Hz}, 1 \mathrm{H}), 7.14(\mathrm{~d}, J=5.0 \mathrm{~Hz}, 1 \mathrm{H})$; ${ }^{13} \mathrm{C}\left\{{ }^{1} \mathrm{H}\right\}$ NMR $\left(100 \mathrm{MHz}, \mathrm{CDCl}_{3}\right) \delta 14.2,15.3,22.8,28.2$, 29.1, 29.7, 31.8, 123.5, 124.0, 126.2, 130.6, 131.4, 133.1, 134.2, 139.8; IR (ATR): 2954, 2926, 2856, 1463, 1199, 1042, 830, 705, $617 \mathrm{~cm}^{-1}$; HRMS (DART-ESI ${ }^{+}$) $\mathrm{m} / \mathrm{z}$ : calcd for $\mathrm{C}_{15} \mathrm{H}_{20}{ }^{35} \mathrm{ClS}_{2}$, 299.0695; found, 299.0687.

2-Chloro-3-methyl-5-(3-(4-(1,1,3,3,3-pentamethyldisiloxy)butan-1-yl)thiophen-2-yl)thiophene (4b) [25]: 72\% yield as a light yellow oil. ${ }^{1} \mathrm{H}$ NMR $\left(400 \mathrm{MHz}, \mathrm{CDCl}_{3}\right) \delta 0.0$ (s, $6 \mathrm{H}), 0.05(\mathrm{~s}, 9 \mathrm{H}), 0.50-0.57(\mathrm{~m}, 2 \mathrm{H}), 1.33-1.44(\mathrm{~m}, 2 \mathrm{H})$, $1.58-1.68(\mathrm{~m}, 2 \mathrm{H}), 2.19(\mathrm{~s}, 3 \mathrm{H}), 2.67-2.74(\mathrm{~m}, 2 \mathrm{H}), 6.77(\mathrm{~s}$, $1 \mathrm{H}), 6.90(\mathrm{~d}, J=5.0 \mathrm{~Hz}, 1 \mathrm{H}), 7.15(\mathrm{~d}, J=5.0 \mathrm{~Hz}, 1 \mathrm{H})$; ${ }^{13} \mathrm{C}\left\{{ }^{1} \mathrm{H}\right\}$ NMR $\left(100 \mathrm{MHz}, \mathrm{CDCl}_{3}\right) \delta 0.5,2.2,13.7,18.4,23.3$, 29.0, 34.5, 124.0, 124.6, 127.6, 128.4, 130.0, 132.7, 134.7, 140.0; IR (ATR): 2955, 2924, 2858, 1567, 1411, 1252, 1194, 1051, 840, 807, 783, 753, 687, 651, $625 \mathrm{~cm}^{-1}$; HRMS (DART$\mathrm{ESI}^{+}$) $\mathrm{m} / \mathrm{z}$ : calcd for $\mathrm{C}_{18} \mathrm{H}_{30}{ }^{35} \mathrm{ClS}_{2} \mathrm{Si}_{2}, 417.0951$; found, 417.0979 .

2-Chloro-3-methyl-5-(3-(4-(bis(trimethylsiloxy)(methyl)silyl)butan-1-yl)thiophen-2-yl)thiophene (4c): 74\% yield as a light yellow oil. ${ }^{1} \mathrm{H} \mathrm{NMR}\left(400 \mathrm{MHz}, \mathrm{CDCl}_{3}\right) \delta 0.0(\mathrm{~s}$, $3 \mathrm{H}), 0.07(\mathrm{~s}, 18 \mathrm{H}), 0.44-0.51(\mathrm{~m}, 2 \mathrm{H}), 1.33-1.42(\mathrm{~m}, 2 \mathrm{H})$, $1.58-1.67(\mathrm{~m}, 2 \mathrm{H}), 2.19(\mathrm{~s}, 3 \mathrm{H}), 2.67-2.73(\mathrm{~m}, 2 \mathrm{H}), 6.77(\mathrm{~s}$, $1 \mathrm{H}), 6.90(\mathrm{~d}, J=5.0 \mathrm{~Hz}, 1 \mathrm{H}), 7.15(\mathrm{~d}, J=5.0 \mathrm{~Hz}, 1 \mathrm{H})$; ${ }^{13} \mathrm{C}\left\{{ }^{1} \mathrm{H}\right\} \mathrm{NMR}\left(100 \mathrm{MHz}, \mathrm{CDCl}_{3}\right) \delta-0.1,2.0,13.7,17.6,23.1$, 28.9, 34.4, 124.1, 124.5, 127.6, 130.0, 130.1, 132.6, 134.7, 140.0; IR (ATR): 2957, 1411, 1256, 1045, 840, 799, 783, 754, 688, $651 \mathrm{~cm}^{-1}$; HRMS (DART-ESI ${ }^{+}$) $\mathrm{m} / \mathrm{z}$ : calcd for $\mathrm{C}_{20} \mathrm{H}_{35}{ }^{35} \mathrm{ClO}_{2} \mathrm{~S}_{2} \mathrm{Si}_{3}$, 491.1153; found, 491.1177 .

2-Chloro-3-(4,4,5,5,6,6,7,7,7-nonafluoroheptyl)-5-(3-(4$(1,1,3,3,3$-pentamethyldisiloxy)butan-1-yl)thiophen-2- yl)thiophene (4d): $46 \%$ yield as a light yellow oil. ${ }^{1} \mathrm{H}$ NMR $\left(400 \mathrm{MHz}, \mathrm{CDCl}_{3}\right) \delta 0.0(\mathrm{~s}, 6 \mathrm{H}), 0.05(\mathrm{~s}, 9 \mathrm{H}), 0.50-0.57(\mathrm{~m}$, $2 \mathrm{H}), 1.33-1.43(\mathrm{~m}, 2 \mathrm{H}), 1.58-1.69(\mathrm{~m}, 2 \mathrm{H}), 1.89-1.99(\mathrm{~m}, 2 \mathrm{H})$, 2.05-2.21 (m, 2H), $2.69(\mathrm{t}, J=7.3 \mathrm{~Hz}, 2 \mathrm{H}), 2.71(\mathrm{t}, J=7.3 \mathrm{~Hz}$, $2 \mathrm{H}), 6.79(\mathrm{~s}, 1 \mathrm{H}), 6.91(\mathrm{~d}, J=5.0 \mathrm{~Hz}, 1 \mathrm{H}), 7.17(\mathrm{~d}, J=5.0 \mathrm{~Hz}$, $1 \mathrm{H}) ;{ }^{13} \mathrm{C}\left\{{ }^{1} \mathrm{H}\right\} \mathrm{NMR}\left(100 \mathrm{MHz}, \mathrm{CDCl}_{3}\right) \delta 0.4,2.1,18.3,20.5$ (br), 23.4, 27.4, 29.0, $30.4(\mathrm{t}, J=22 \mathrm{~Hz}), 34.5,124.3,125.1$, 126.0, 129.7, 130.2, 133.6, 137.6, 140.3; ${ }^{19} \mathrm{~F}$ NMR (376 MHz, $\left.\mathrm{C}_{6} \mathrm{~F}_{6}\right) \delta-129.2,-127.6,-117.6,-84.2$; IR (ATR): 2957, 1252, 1232, 1167, 1133, 1101, 1057, 1013, 879, 842, 807, 784, 752, 736, 719, 689, $651 \mathrm{~cm}^{-1}$; HRMS (DART-ESI ${ }^{+}$) $\mathrm{m} / \mathrm{z}$ : calcd for $\mathrm{C}_{24} \mathrm{H}_{33}{ }^{35} \mathrm{ClF}_{9} \mathrm{OS}_{2} \mathrm{Si}_{2}$, 663.1056; found, 663.1050.

2-Chloro-3-(4,4,5,5,6,6,7,7,7-nonafluoroheptyl)-5-(3-(4(bis(trimethylsiloxy)(methyl)silyl)butan-1-yl)thiophen-2yl)thiophene (4e): $50 \%$ yield as a light yellow oil. ${ }^{1} \mathrm{H}$ NMR $\left(400 \mathrm{MHz}, \mathrm{CDCl}_{3}\right) \delta 0.0(\mathrm{~s}, 3 \mathrm{H}), 0.07(\mathrm{~s}, 18 \mathrm{H}), 0.44-0.52(\mathrm{~m}$, $2 \mathrm{H}), 1.32-1.43(\mathrm{~m}, 2 \mathrm{H}), 1.58-1.68(\mathrm{~m}, 2 \mathrm{H}), 1.89-1.99(\mathrm{~m}, 2 \mathrm{H})$, $2.04-2.21(\mathrm{~m}, 2 \mathrm{H}), 2.68(\mathrm{t}, J=7.8 \mathrm{~Hz}, 2 \mathrm{H}), 2.70(\mathrm{t}, J=7.8 \mathrm{~Hz}$, $2 \mathrm{H}), 6.79(\mathrm{~s}, 1 \mathrm{H}), 6.91(\mathrm{~d}, J=5.0 \mathrm{~Hz}, 1 \mathrm{H}), 7.17(\mathrm{~d}, J=5.0 \mathrm{~Hz}$, $1 \mathrm{H}) ;{ }^{13} \mathrm{C}\left\{{ }^{1} \mathrm{H}\right\} \mathrm{NMR}\left(100 \mathrm{MHz}, \mathrm{CDCl}_{3}\right) \delta-0.2,2.0,17.6,20.5$ (br), 23.1, 27.4, 29.0, 30.4 (t, $J=22 \mathrm{~Hz}), 34.3,124.3,125.1$, 126.0, 129.7, 130.2, 133.6, 137.6, 140.2; ${ }^{19} \mathrm{~F}$ NMR (376 MHz, $\left.\mathrm{C}_{6} \mathrm{~F}_{6}\right) \delta-129.2,-127.6,-117.6,-84.2$; IR (ATR): 2958, 1252, 1233, 1167, 1134, 1046, 870, 841, 800, 783, 754, 719, 689, $651 \mathrm{~cm}^{-1}$; HRMS (DART-ESI ${ }^{+}$) $\mathrm{m} / \mathrm{z}$ : calcd for $\mathrm{C}_{27} \mathrm{H}_{43}{ }^{35} \mathrm{ClF}_{9} \mathrm{OS}_{2} \mathrm{Si}_{3}$, 737.1608; found, 737.1611.

General procedure for the polymerization of chlorobithiophene representatives: the reaction of $4 \mathrm{~b}$ leading to poly(3(4-(1,1,3,3,3-pentamethyldisiloxy)butan-3-yl)thiophen-2,5diyl)-alt-poly(3-methylthiophen-2,5-diyl) (6b): To a $20 \mathrm{~mL}$ Schlenk tube equipped with a magnetic stirring bar were added 4b (104 mg, $0.25 \mathrm{mmol})$ and a $1 \mathrm{M}$ THF solution of $\mathrm{TMPMgCl} \cdot \mathrm{LiCl}(0.3 \mathrm{~mL}, 0.3 \mathrm{mmol})$ was added at room temperature. After stirring at room temperature for $3 \mathrm{~h}$, THF $(2.5 \mathrm{~mL})$ and $\mathrm{NiCl}_{2}\left(\mathrm{PPh}_{3}\right) \operatorname{IPr}(5,3.9 \mathrm{mg}, 6.0 \mu \mathrm{mol})$ were added to initiate the polymerization. The color of the solution changed to light orange and then to dark purple with the formation of slightly insoluble material. After stirring at room temperature for $24 \mathrm{~h}$, the reaction mixture was poured into a mixture of hydrochloric acid $(1.0 \mathrm{M}, 2 \mathrm{~mL})$ and methanol $(10 \mathrm{~mL})$ to form a precipitate, which was filtered off to leave a dark purple solid. After washing with methanol and hexanes repeatedly, the solid was dried under reduced pressure to afford $79.6 \mathrm{mg}$ of $\mathbf{6 b}(84 \%$ isolated yield). The HT regioregularity was confirmed by ${ }^{1} \mathrm{H}$ NMR analysis, and the molecular weight $\left(M_{\mathrm{n}}\right)$ and the molecular weight distribution $\left(M_{\mathrm{w}} / M_{\mathrm{n}}\right)$ were estimated by SEC analysis. $\mathrm{HT}=98 \%, M_{\mathrm{n}}=10400, M_{\mathrm{w}}=16900, M_{\mathrm{w}} / M_{\mathrm{n}}=1.62$; ${ }^{1} \mathrm{H}$ NMR (400 MHz, $\mathrm{CDCl}_{3}$ ) $\delta$ 0.02-0.08 (br, 15H), 0.50-0.63 $(\mathrm{m}, 2 \mathrm{H}), 1.41-1.52(\mathrm{~m}, 2 \mathrm{H}), 1.64-1.78(\mathrm{~m}, 2 \mathrm{H}), 2.44(\mathrm{~s}, 3 \mathrm{H})$, 
2.75-2.85 (m, 2H), $6.95(\mathrm{~s}, 1 \mathrm{H}), 6.99(\mathrm{~s}, 1 \mathrm{H})$; IR (ATR): 2956, 2925, 2855, 1728, 1445, 1252, 1057, 841, 806, 782, $753 \mathrm{~cm}^{-1}$.

The other polymers $\mathbf{6 c}-\mathbf{e}$ were synthesized similarly. The polymerization was continued for 18-24 h or terminated after $1 \mathrm{~h}$ when $\mathbf{4}$ bearing a fluoroalkyl substituent was employed, due to faster polymerization, which brought about an uncontrollable molecular weight. The properties and spectroscopic data are summarized below.

Poly(3-(4-(bis(trimethylsiloxy)(methyl)silyl)butan-1-yl)thiophen-2,5-diyl)-alt-poly(3-methylthiophen-2,5-diyl) (6c): $84 \%$ yield. $M_{\mathrm{n}}=70100, M_{\mathrm{w}} / M_{\mathrm{n}}=1.38 ;{ }^{1} \mathrm{H} \mathrm{NMR}\left(400 \mathrm{MHz}, \mathrm{CDCl}_{3}\right)$ $\delta 0.03(\mathrm{~s}, 3 \mathrm{H}), 0.09(\mathrm{~s}, 18 \mathrm{H}), 0.43-0.65(\mathrm{~m}, 2 \mathrm{H}), 1.35-1.55(\mathrm{~m}$, $2 \mathrm{H}), 1.63-1.85(\mathrm{~m}, 2 \mathrm{H}), 2.44(\mathrm{~s}, 3 \mathrm{H}), 2.71-2.92(\mathrm{~m}, 2 \mathrm{H}), 6.95$ $(\mathrm{s}, 1 \mathrm{H}), 7.00(\mathrm{~s}, 1 \mathrm{H}) ;{ }^{13} \mathrm{C}\left\{{ }^{1} \mathrm{H}\right\}$ NMR $\left(100 \mathrm{MHz}, \mathrm{CDCl}_{3}\right) \delta-0.1$, 2.1, 15.9, 17.7, 23.3, 29.4, 34.2, 128.3, 130.0, 130.4, 131.0, 133.5, 134.3, 134.4, 140.0; IR (ATR): 2957, 2926, 2858, 1511, $1449,1256,1046,841,802,782,754,668 \mathrm{~cm}^{-1}$.

Poly(3-(4-(1,1,3,3,3-pentamethyldisiloxy)butan-3-yl)thiophen-2,5-diyl)-alt-poly (3-(4,4,5,5,6,6,7,7,7-nonafluoroheptyl)thiophen-2,5-diyl) (6d): $48 \%$ yield. $M_{\mathrm{n}}=68600$, $M_{\mathrm{w}} / M_{\mathrm{n}}=1.15 ;{ }^{1} \mathrm{H} \mathrm{NMR}\left(400 \mathrm{MHz}, \mathrm{CDCl}_{3}\right) \delta 0.06(\mathrm{~s}, 15 \mathrm{H})$, 0.55-0.66 (m, 2H), 1.41-1.55 (m, 2H), 1.67-1.83 (m, 2H), $1.97-2.12(\mathrm{~m}, 2 \mathrm{H}), 2.12-2.30(\mathrm{~m}, 2 \mathrm{H}), 2.73-2.88(\mathrm{~m}, 2 \mathrm{H})$, 2.88-3.01 (m, 2H), $7.00(\mathrm{br}, 2 \mathrm{H}) ;{ }^{13} \mathrm{C}\left\{{ }^{1} \mathrm{H}\right\}$ NMR (100 MHz, $\left.\mathrm{CDCl}_{3}\right) \delta 0.5,2.1,18.4,21.3(\mathrm{br}), 23.5,28.7,29.4,30.6$ (t, $J=$ $22 \mathrm{~Hz}), 34.4,128.2$, 129.3, 130.7, 131.6, 133.3, 134.4, 137.8, 140.4; IR (ATR): 2957, 2925, 1452, 1356, 1252, 1232, 1169, $1134,1059,879,842,807,783,752,720,701 \mathrm{~cm}^{-1}$.

Poly(3-(4-(bis(trimethylsiloxy)(methyl)silyl)butan-1-yl)thiophen-2,5-diyl)-alt-poly (3-(4,4,5,5,6,6,7,7,7-nonafluoroheptyl)thiophen-2,5-diyl) (6e): 58\% yield. SEC analysis showed $M_{\mathrm{n}}=33200, M_{\mathrm{w}} / M_{\mathrm{n}}=1.07 ;{ }^{1} \mathrm{H} \mathrm{NMR}(400 \mathrm{MHz}$, $\left.\mathrm{CDCl}_{3}\right) \delta 0.03(\mathrm{~s}, 3 \mathrm{H}), 0.09(\mathrm{~s}, 18 \mathrm{H}), 0.44-0.66(\mathrm{~m}, 2 \mathrm{H})$, $1.37-1.60(\mathrm{~m}, 2 \mathrm{H}), 1.60-1.86(\mathrm{~m}, 2 \mathrm{H}), 1.94-2.33(\mathrm{~m}, 4 \mathrm{H})$, 2.63-3.11 (m, 4H), $7.00(\mathrm{br}, 2 \mathrm{H}) ;{ }^{13} \mathrm{C}\left\{{ }^{1} \mathrm{H}\right\}$ NMR $(100 \mathrm{MHz}$, $\left.\mathrm{CDCl}_{3}\right) \delta-0.2,2.0,17.7,21.3,23.3,28.7,29.4,30.6$ (t, $J=22$ $\mathrm{Hz}), 34.2$, 128.2, 129.3, 130.7, 131.6, 133.3, 134.5, 137.8, 140.4; IR (ATR): 2957, 1455, 1356, 1251, 1233, 1134, 1052, $840,801,773,754,720,700 \mathrm{~cm}^{-1}$.

\section{Funding}

This work was partially supported by JSPS Kakenhi B Grant Number JP19182273, Cooperative Research Program of "Network Joint Research Center for Materials and Devices", and Kobe University for the promotion of international collaboration researches.

\section{ORCID ${ }^{\circledR} \mathrm{iDs}$}

Atsunori Mori - https://orcid.org/0000-0002-1163-264X Masaki Horie - https://orcid.org/0000-0002-7734-5694

\section{Preprint}

A non-peer-reviewed version of this article has been previously published as a preprint doi:10.3762/bxiv.2019.160.v1

\section{References}

1. Sirringhaus, H.; Tessler, N.; Friend, R. H. Science 1998, 280 , 1741-1744. doi:10.1126/science.280.5370.1741

2. Li, G.; Shrotriya, V.; Huang, J.; Yao, Y.; Moriarty, T.; Emery, K.; Yang, Y. Nat. Mater. 2005, 4, 864-868. doi:10.1038/nmat1500

3. Osaka, I.; McCullough, R. D. Acc. Chem. Res. 2008, 41, 1202-1214. doi:10.1021/ar800130s

4. Bao, Z.; Dodabalapur, A.; Lovinger, A. J. Appl. Phys. Lett. 1996, 69, 4108-4110. doi:10.1063/1.117834

5. Yokozawa, T.; Ohta, Y. Chem. Rev. 2016, 116, 1950-1968. doi:10.1021/acs.chemrev.5b00393

6. Okamoto, K.; Luscombe, C. K. Polym. Chem. 2011, 2, 2424-2434. doi:10.1039/c1py00171j

7. Krasovskiy, A.; Krasovskaya, V.; Knochel, P. Angew. Chem., Int. Ed. 2006, 45, 2958-2961. doi:10.1002/anie.200504024

8. Tamba, S.; Shono, K.; Sugie, A.; Mori, A. J. Am. Chem. Soc. 2011, 133, 9700-9703. doi:10.1021/ja2033525

9. Tamba, S.; Tanaka, S.; Okubo, Y.; Meguro, H.; Okamoto, S.; Mori, A Chem. Lett. 2011, 40, 398-399. doi:10.1246/cl.2011.398

10. Matsubara, K.; Ueno, K.; Shibata, Y. Organometallics 2006, 25 , 3422-3427. doi:10.1021/om0602658

11. Herrmann, W. A. Angew. Chem., Int. Ed. 2002, 41, 1290-1309. doi:10.1002/1521-3773(20020415)41:8<1290::aid-anie1290>3.0.co;2-y

12. Fujita, K.; Sumino, Y.; Ide, K.; Tamba, S.; Shono, K.; Shen, J.; Nishino, T.; Mori, A.; Yasuda, T. Macromolecules 2016, 49, 1259-1269. doi:10.1021/acs.macromol.5b02524

13. Mori, A.; Ide, K.; Tamba, S.; Tsuji, S.; Toyomori, Y.; Yasuda, T. Chem. Lett. 2014, 43, 640-642. doi:10.1246/cl.131222

14. Ogura, T.; Kubota, C.; Suzuki, T.; Okano, K.; Tanaka, N.; Matsumoto, T.; Nishino, T.; Mori, A.; Okita, T.; Funahashi, M. Chem. Lett. 2019, 48, 611-614. doi:10.1246/cl.190139

15. Noh, S.; Gobalasingham, N. S.; Thompson, B. C. Macromolecules 2016, 49, 6835-6845. doi:10.1021/acs.macromol.6b01178

16. Locke, J. R.; McNeil, A. J. Macromolecules 2010, 43, 8709-8710. doi:10.1021/ma102218y

17. Palermo, E. F.; Darling, S. B.; McNeil, A. J. J. Mater. Chem. C 2014, 2, 3401-3406. doi:10.1039/c3tc32512a

18. Lee, E.; Hammer, B.; Kim, J.-K.; Page, Z.; Emrick, T.; Hayward, R. C. J. Am. Chem. Soc. 2011, 133, 10390-10393. doi:10.1021/ja2038547

19. Ouhib, F.; Desbief, S.; Lazzaroni, R.; De Winter, J.; Gerbaux, P.; Jérôme, C.; Detrembleur, C. Macromolecules 2012, 45, 6796-6806. doi:10.1021/ma3009405

20. Hong, X. M.; Tyson, J. C.; Collard, D. M. Macromolecules 2000, 33, 3502-3504. doi:10.1021/ma991997x

21. Bjørnholm, T.; Greve, D. R.; Reitzel, N.; Hassenkam, T.; Kjaer, K.; Howes, P. B.; Larsen, N. B.; Bøgelund, J.; Jayaraman, M.; Ewbank, P. C.; McCullough, R. D. J. Am. Chem. Soc. 1998, 120 , 7643-7644. doi:10.1021/ja981077e 
22. Reitzel, N.; Greve, D. R.; Kjaer, K.; Howes, P. B.; Jayaraman, M.; Savoy, S.; McCullough, R. D.; McDevitt, J. T.; Bjørnholm, T. J. Am. Chem. Soc. 2000, 122, 5788-5800. doi:10.1021/ja9924501

23. Smith, Z. C.; Meyer, D. M.; Simon, M. G.; Staii, C.; Shukla, D.; Thomas, S. W., III. Macromolecules 2015, 48, 959-966. doi:10.1021/ma502289n

24. Nakamura, K.; Tamba, S.; Sugie, A.; Mori, A. Chem. Lett. 2013, 42, 1200-1202. doi:10.1246/cl.130523

25. Fujita, K.; Nakagawa, N.; Sunahara, K.; Ogura, T.; Okano, K.; Mori, A. Synthesis 2017, 49, 1285-1294. doi:10.1055/s-0036-1588094

26. Kantchev, E. A. B.; O'Brien, C. J.; Organ, M. G. Angew. Chem., Int. Ed. 2007, 46, 2768-2813. doi:10.1002/anie.200601663

27. Sun, D.; Li, Y.; Ren, Z.; Bryce, M. R.; Li, H.; Yan, S. Chem. Sci. 2014, 5, 3240-3245. doi:10.1039/c4sc01068j

28. Nakagawa, N.; Ogura, T.; Fujita, K.; Sumino, Y.; Hashimoto, T.; Okano, K.; Mori, A. Chem. Lett. 2017, 46, 453-455. doi:10.1246/cl.161180

29. Shen, J.; Fujita, K.; Matsumoto, T.; Hongo, C.; Misaki, M.; Ishida, K.; Mori, A.; Nishino, T. Macromol. Chem. Phys. 2017, 218, No. 1700197. doi:10.1002/macp.201700197

30. Matsumoto, T.; Nishi, K.; Tamba, S.; Kotera, M.; Hongo, C.; Mori, A.; Nishino, T. Polymer 2017, 119, 76-82. doi:10.1016/j.polymer.2017.05.027

31. Inoue, R.; Yamaguchi, M.; Murakami, Y.; Okano, K.; Mori, A. ACS Omega 2018, 3, 12703-12706. doi:10.1021/acsomega.8b01707

\section{License and Terms}

This is an Open Access article under the terms of the Creative Commons Attribution License (https://creativecommons.org/licenses/by/4.0). Please note that the reuse, redistribution and reproduction in particular requires that the authors and source are credited.

The license is subject to the Beilstein Journal of Organic Chemistry terms and conditions:

(https://www.beilstein-journals.org/bjoc)

The definitive version of this article is the electronic one which can be found at: $\underline{\text { doi:10.3762/bjoc. } 16.31}$ 